\title{
AUTOMATIC MODELLING OF BUILDING FAÇADE OBJECTS VIA PRIMITIVE SHAPES
}

\author{
Hetti Arachchige Nalani ${ }^{a}$,,$*$, Sanka Perera ${ }^{b}$ \\ ${ }^{a}$ Faculty of Geomatics, Sabaragamuwa University of Sri Lanka, 70140, Belihuloya, Sri Lanka, \\ ${ }^{\mathrm{b}}$ Institute of Photogrammetry and Remote Sensing, Dresden University of Technology, \\ nalani@sab.ac.lk
}

Commission III

KEY WORDS: Detection, Mobile Laser Scanners, Point clouds, Geometric Feature, Urban Trees, Principal direction

\begin{abstract}
:
This paper presents a new approach to recognize individual façade objects and to reconstruct such objects in 3D using MLS point clouds. Core of the approach is a primitive shape based algorithm, which introduces building primitives, to identify the façade objects separately from other irrelevant objects and then to model the correct topology. The primitive shape is identified against defined different primitive shapes by using the Douglas-Peucker algorithm. The advantage of this process is that it offers an ability not only to model correct geometric shapes but also to remove occlusion effects from the final model. To evaluate the validity of the proposed approach, experiments have been conducted using two types of street scene point clouds captured by Optech Lynx Mobile Mapper System and Z+F laser scanner. Results of the experiments show that the completeness, correctness, and quality of the reconstructed building façade objects are well over $90 \%$, proving the proposed method is a promising solution for modelling 3D façade objects with different geometric shapes.
\end{abstract}

\section{INTRODUCTION}

3D building façade models have become essential to many applications related to human activities in urban areas such as urban planning, autonomous navigation, tourism, telecommunication, virtual $3 \mathrm{D}$ modelling and so on. This essentiality's interest has been increased, rapidly with the development of laser scanning technology offering capability to automatically reconstruct high level of detail models of building facades such as LoD2 or LoD3. Therefore, the digital recording of urban building facades using laser scanning has become a topic of great interest especially in the field of urban management and tourism. In the recent past, mobile laser scanning (MLS) has proven to be an invaluable technique to acquire complex 3D geometry of real world objects in large areas.

In many cases, a point cloud acquired from laser scanner is used as the final mesh models, as such analysis and application of those models is restricted primarily to visualization and some basic measurements. The reconstruct of geometric models lets for various applications, for example, semantic modelling, which enables not only visualization of data but also complex analysis, and managements. However, due to the high complexity of building façade structures, urban scenes, and also due to the occlusion effects, no fully automatic methods for reconstructing accurate geometric façade models are available. Further only few efforts on façade modelling have been developed. Most of them focus only on particular shape façade objects, and relatively simple rectangular shape ones. The approach presented in this paper uses an automatic technique to recognize individual façade objects and to model such objects in 3D from MLS point cloud.
Man-made objects differ from each other according to their geometric shape. These geometric shapes can be described using various primitive shapes. As such, façade objects can easily be classified from other irrelevant objects and then can be obtained the correct geometrical models. The methodology uses primitive shapes to describe each façade feature. These primitive shapes can generally be defined based on building architectural (shape) knowledge.

The proposed method can work on raw point clouds even with a significant amount of noise. First, a local height histogram is used to filter out ground points. The process starts with segmenting the non-ground points into planar surfaces corresponding to the façade objects. We then recognized basic façade objects such as walls, roofs and windows in a segmented laser point cloud. An angle based criteria is used to identify boundary points of each object. As part of that, a neighbourhood is defined based on the triangular irregular network (TIN) while removing long TIN edges. Opening areas in the wall facades are then assessed by comparing their characteristics to those of real façade objects. In modelling process, we introduced a new concept to model these objects by minimizing occlusion effects. For this, we assume that each façade object has two basic components: lower and upper components. Lower part consists of a rectangular shape part or a union of rectangular shape parts. Accordingly only horizontal and vertical line segments are extracted from boundary points of the lower part. For this, a histogram analysis method is used. The advantage of this process is that it offers an ability not only to model correct geometric shape but also to remove occlusion effects from the final model. A complete model relevant to lower part is generated by employing the geometric constraints. Then the upper shape is searched according to the defined different primitive shapes such as circular, triangular, linear and

\footnotetext{
* Corresponding author.
} 
so on. To cope with this, the Douglas-Peucker algorithm, known from boundary generalization theory, is introduced to correctly identify the primitive shapes of upper part. The shape knowledge is again used as constraints to obtain a complete facade model with a more realistic view. To maintain the symmetry, mirror concept of the left-side is utilized.

The overall structure of the paper is organized as follows: the next section gives a review of previous literature on building façade reconstruction from terrestrial point cloud data. In section 3 and 4 , the proposed method is discussed. Section 5 comprises the evaluation of the method and discussion. The paper is concluded in section 6 .

\section{RELATED WORKS}

Façade modelling based on geometric primitives start by segmenting the point clouds into homogeneous point groups. These segments are used to recognize façade objects. The segmentation of laser scanner point clouds can be considered as a first step in the working scheme of building facade reconstruction process. It could be followed by geometric modelling thereafter. During the last decade, only few contributions have been made using point cloud data.

Approaches using images especially for window reconstruction have been proposed. In most of these works, object edge detection is accomplished on the façade texture (Reznik and Mayer, 2008; Meixner and Leberl, 2010). Regarding point cloud data, Briese et al. (2008) propose a semi-automatic reconstruction technique using linear features extracted from Terrestrial Laser Scanning (TLS) data. To overcome difficulties of methods which are exclusively based on a point clouds, Haala et al., (2006), Böhm et al. (2007) and Pu and Vosselman. (2009) present a façade modelling method by combining point clouds and photographs in a semi-automatic way.

Most of the reconstruction methods do not consider semantic information into reconstruction process. The object shape knowledge on the building facades and of its components is most important in the case of data gaps. Ripperda (2008) presents grammar rules for façade object parameters which are solely from image data. Another interesting method has been proposed by Becker (2009) using formal grammars to describe façade elements. The grammars are derived from TLS point data and refinement is done with image data. With the help of the formal grammars, building objects, which are occluded or missed in the data, can be modelled.

Boulaassal et al. (2009) use solely point clouds. It is based on a 2D Delaunay triangulation of the segmented façade plane. In this process, the point data segment into planar surfaces by using sequential RANSAC. Since windows can often be described as holes in the planar surface, triangles corresponding to windows are identified by looking for long triangles than a defined threshold. The vertices of the selected triangles are considered as edge points of the object. Those points have been used for creating polygons representing of complete models by transforming them in to parametric model. Based on these contours, the CAD models are constructed. Similar point cloud method is presented by $\mathrm{Pu}$ and Vosselman (2009). Different constraints defined based on building knowledge are used to extract façade features from a segmented planar surfaces. Assuming a homogenous point density on the selected façade plane, windows are identified by searching for no measurement areas in the plane. To reconstruct geometric models, a minimum bounding rectangle is fitted to each hole found on the wall plane by using a least-square line fitting approach. Some drawbacks comprise low accuracy outlines, incomplete window generation, and dependence on a defined distance threshold. Schmittwilken and Plümer (2010) propose an approach based on prior knowledge from training data. Different parameters to describe shape of windows and stairs are used for the selection of the sample for each tested object.

the proposed approach aims to utilize the primitives for describe geometric shape of each façade objects. Most of the existing method result rectangular shape windows and doors, the presented approach could be used to model not only rectangular shapes but also different shape windows. The method is solely based on point clouds acquired by MLS.

\section{SEGMENTATION AND OBJECT EXTRACTION}

An initial processing step is performed to classify points into two groups: ground and object points. The non-ground points serve as fundamental input for the recognition of urban objects, i.e. building façades. Ground points can generally be defined as the points residing on lowest and smooth horizontal surface. Thus, filtering of ground points was performed by combining the local height histogram analysis method, similar to the method described by Maas at al. (2008) to generate a TLSbased terrain model, and a planar surface growing method.

The first task of the façade reconstruction process is the segmentation, in which object points segment into disjoined point groups to identify façade objects. A shape-based method for the segmentation of large-scale urban point clouds is proposed. Procedure of the method consists of three sequential stages: linear feature extraction; point classification based on their geometric features (surface roughness) and planar segmentation and merging of these segments to archive correct geometric abstraction of façade features. In the planar segmentation, surface roughness based region growing method is applied. After segmenting points into planar surfaces, object recognition step is performed. A feature recognition based on geometric constraints derived from knowledge-based is applied. More details to these processes can be seen in Nalani et al. (2012).

\section{GEOMETRIC MODEL RECONSTRUCTION}

\subsection{Window/door object recognition}

Recognized wall segments could generally be explained by vertical planar surfaces with openings comprising windows or doors (structural elements residing within a wall façade). As explained by $\mathrm{Pu}$ and Vosselman (2007) and Becker and Haala (2007), window/door objects are found by observing opening areas (holes) on the detected wall façades.

Whole process of the applied hole-based feature detection scheme depicted in Figure 1 could be divided into three stages: (I) boundary point extraction, (II) individual object identification, i.e. clustering boundary points on the same opening, and (III) filtering of unrealistic objects. The following Sections elaborate each of these step in detail. 


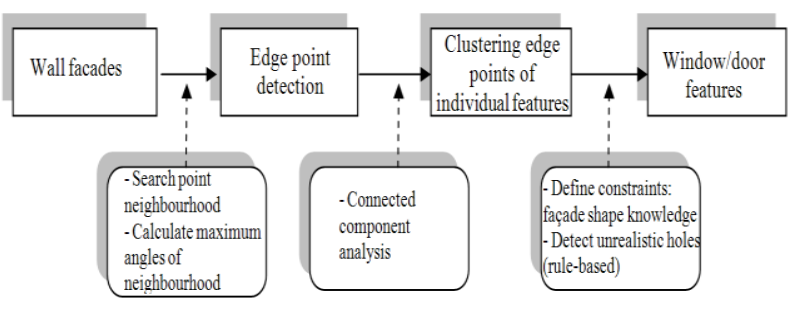

Figure 1: Workflow of façade object detection

Let $W$ be a building wall surface including the set of points $P_{i}=\left\{p_{1}, \ldots, p_{n}\right\} \subset \mathfrak{R}^{3}$. The feature detection can then be defined as $E_{P}(P) \rightarrow\{p \in P \mid p \quad$ is edge $\}$ that recognises the set of edge points $E=E_{P}(P)$ circumscribing holes in $P$.

In order to identify boundary points, different methods and criteria such as the Delaunay triangulation, the half-disc method and the voxelization method (Becker and Haala, 2007; Boulaassal et al., 2009; $\mathrm{Pu}, 2010$ ) have been utilized. The angle criterion is more consistent in classifying corner points and extracting sharp and thin edges compared to other methods (Bendels et al., 2006). This work introduces a technique through the combination of Delaunay triangulation and an angle criterion, which can harvest greater number of edge points correctly.

First, 3D points corresponding to a façade wall are transformed into a façade planar coordinate system with the $X$ and $Z$-axis being parallel to the horizontal and vertical direction of the plane respectively. The process of boundary point detection starts with a randomly chosen point $\left(p_{i}\right)$ in the wall point set and then finds neighbouring points of $p_{i}\left(: q_{i}\right.$ where $i=i \ldots n$ and $j=i \ldots m)$. The given point confirmed as boundary point if the angle $\left(\theta_{i, i+1}\right)$ between two consecutive neighbour points, sorted according to their azimuth, exceeds the maximum angle threshold $\left(\alpha=<\theta_{j, j+1}\right)$, as depicted in Figure 2 (a). Otherwise, the point is rejected as a wall interior point. The threshold for the angle is adopted as $\pi / 2^{\circ}$ to select most of edge points and not to be misclassified corner points. The criterion is continued till all points on the façade wall classified either as interior or edge point.
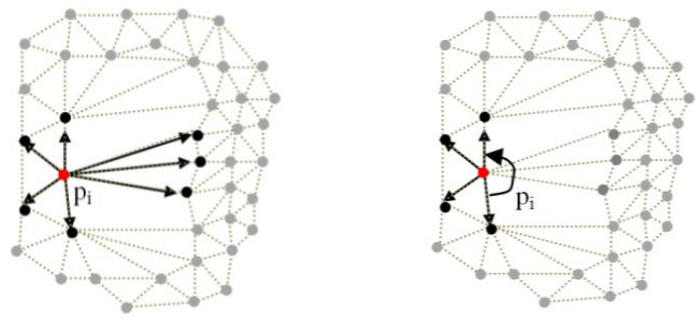

Figure 2: Boundary point detection: (a) neighbour point distribution of the edge point $P_{i}$ (points indicating arrows) with TNN neighbourhood; and (b) after removing long TIN edges.

Detected boundary points are clustered using a connected component analysis technique, as described by $\mathrm{Pu}$ (2010) to identify holes representing individual objects. It considers TIN edges except long TIN edges instead of using only long TIN edges. The boundary points of objects located closer to the ground are unable to detect. This is because the lower part of the holes is opened and their boundaries are connected to the façade wall. In such cases, objects are isolated by analysing wall-contour points. It consists of the wall-contour creation followed by the searching of concave bended parts on the wallcontour based on height differences between neighbouring points of the contour. Only lower part of the wall-contour is considered and updated by removing the selected parts.

There are some holes caused by occlusion effects. According to the architectural rules, doors and windows have their own standard shapes and sizes. To retrieval actual holes representing the façade features, rules describing geometric shape and neighbour relations are defined. For example, a cue can be written as:

$$
f(h, w, A)= \begin{cases}h_{\text {ole }} \text { facade } & \text { if }\left(h>h_{\min } \text { and } w>w_{\min }\right) \text { or }\left(A<A_{\min }\right) \\ \text { hole } e_{\text {noise }} & \text { else }\end{cases}
$$

Where, $h, w$, and $A$ are geometric parameters: height, width and area respectively.

Nevertheless, some of selected objects are partly affected by occlusions or other neighbouring objects. Also, some other objects do not belong to an actual façade object as a result of large holes caused by occlusion. Generally, shape of most of façade objects is rectangular or that have rectangular shape bases. The shape can be defined as a union of primitive shapes including rectangular shape primitives. These rectangular shape parts can be represented by using line and point primitives. As such, each object should have at least one vertical line $(V)$. Therefore, an edge orientation step is employed to distinguish façade objects from others. The cue can be written as:

$$
f(V)=\left\{\begin{array}{l}
\text { hole }_{\text {facade }} \text { if }\left(V_{\text {line }}>0\right) \\
\text { hole }_{\text {noise }} \text { else }
\end{array}\right.
$$

A more detail explanation is given in the Section 4.2 as the process is applied during the model reconstruction process.

\subsection{Geometric fitting via primitive shapes}

As explained in Section 4.1, a façade object can generally be modelled by combining several primitive shapes such as rectangular boxes, cylinders and cone. Three basic primitives: namely point, line and plane, are used to describe each primitive shape. Therefore, basic primitives corresponding to each object should be extracted first and connected to approximate the geometry of the object the shape can be generalized as upper and lower shape. Based on this, boundary points of each object are divided in to two groups; upper and lower. Accordingly, geometric model of an object is reconstructed considering lower and upper part separately.

Both vertical and horizontal lines representing object boundaries are extracted using a histogram analysis technique. To find vertical lines, a histogram along $Y$ direction, i.e., a number of bins in $Y$ direction is constructed and fitted with a sequence of line segments for the edge points corresponding to the selected bins (Pighin and Lewis, 2007). Based on this, points related to the lower part are found. The horizontal lines from boundary points in the lower part are extracted. This is demonstrated in Figure 3. It consists of two rectangles referring to the lower part and a triangular shape upper part. 


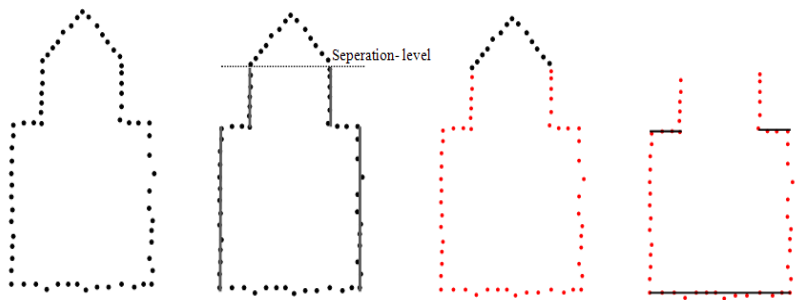

Figure 3: Geometric fitting: (a) boundary points; (b) extracted vertical line segments; (c) separated into two parts; and (d) extracted horizontal line segments

By analysing the proximity between end points of each line segment, the line-adjacency list is created to get sequentially ordered line segments. With help of the line adjacency, geometry (a polyline) of the lower part is generated. Orthogonality between neighbouring lines is searched during the model fitting. Accordingly, new lines are inserted to form a complete model.

Boundary points above the separation-level (as shown in Figure 3) are chosen as points corresponding to the upper part of the object. Real shape of the upper part could often be represented by straight (rectangular shape), curve (arched shape) or bilinear (wedge shape) line primitives. To discover the actual shape of the upper part, a method similar to the Douglas-Peucker (Douglas and Peucker, 1973), which identifies turning points where the large changes occurred, is applied. The actual shape of the feature is then defined according to the number of turning points. For example, if there are two turning points, it is assumed that the upper part has a linear shape. Three turning points indicate triangular shape while more points represent either a circular or an irregular shape.

After identifying the shape, a poly-line referring to the upper part is constructed by connecting consecutive turning points if the upper shape is rectangular, triangular or irregular. In the case of circular shapes, new edge nodes have to be inserted with respect to the least-square circle fitting.

\section{RESULTS AND DISCUSSION}

To validate the presented algorithm, two data sets have been experimented. Experimental tests are performed to assess benefits and limitations of the developed approach. The first data set is located in the city of Dresden, Germany. The dataset has been acquired by a $\mathrm{Z}+\mathrm{F}$ laser scanner with a $310^{\circ} \times 360^{\circ}$ field of view and $0.1 \mathrm{~mm}$ range resolution. The second data set has been acquired by TopScan GmbH using the Optech Lynx mobile mapping system. It is a scene from an urban area located in Bonn, Germany.

Several experiments are performed on these two datasets. The visual appearances of the obtained results for some of selected building facades (walls) from each dataset are shown in Figure 4, 5, 6 and 7. To evaluate the performance of the presented algorithm, interactively estimated statistics have been used as a benchmark.

A result obtained from test on a simple wall façade comprising 15 rectangular shape windows with two different sizes is shown in Figure 4. A planar surface (Figure $4 \mathrm{~b}$ ) is selected as a wall from segmented point cloud of the test area. Boundary points
(Figure 4c) of the wall façade are extracted using the anglebased method and the 3D façade models. All façade objects (Figure 4d) are reconstructed by connecting line segments, which are extracted using the detected edge points. A visual comparison with the respective photograph shows that all windows of the wall have been reconstructed with correct topological relations. The recovered structure satisfies the requirements of LOD2. Some parts of the wall surface, especially the lower part, have been occluded by other surfaces closer to the building wall such as vegetation and vehicles. Although such areas have been selected as façade objects during the process of boundary point clustering (two clusters in the black boxes), they have been eliminated from the reconstructed results. Total runtime for the wall has been around 10 seconds.
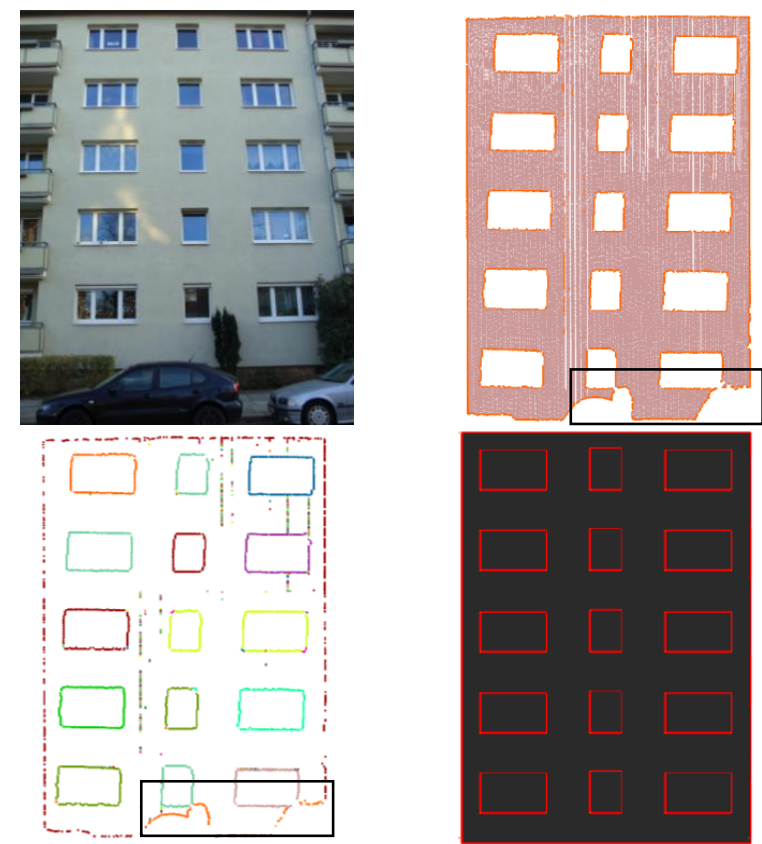

Figure 1: Results of façade reconstruction process for a Wall in area1: (a) a photograph of the relevant wall facade; (b) detected edge points (red); (c) detected individual objects; and (d) the geometric model.

Figure 5 shows another wall where the developed algorithm giving satisfying results. When the reconstructed results have been visually compared with the reference photograph, It cloud be seen that the algorithm is worked well not only on rectangular shape objects but also on other-shaped objects. Some small and narrow holes caused by occlusion effect (red/yellow arrows) have been filtered by using geometric constraints. 

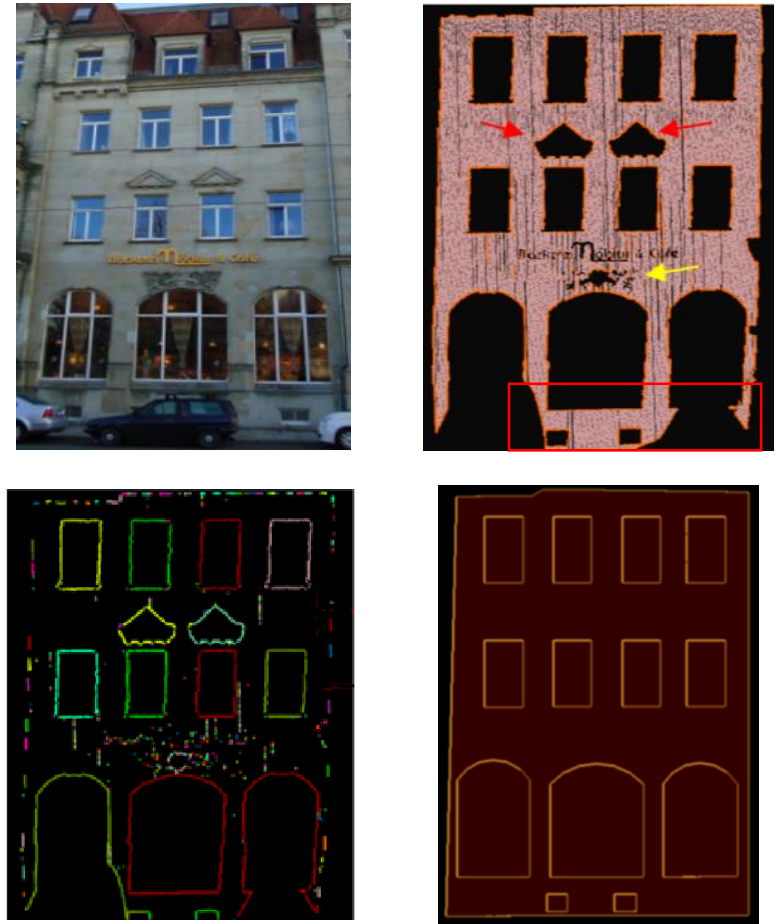

Figure 2: Results of façade reconstruction process for a selected wall: (a) a photograph; (b) detected edge points; (c) extracted clusters; and (d) final model

Another successful modelling result of selected walls is shown in Figure 6. More than $80 \%$ of façade information is available in the final façade models. These experimental results demonstrate the high potential of the proposed method. A statistical analysis on detected and reconstructed façade features for each dataset is presented in the next section.

A qualitative assessment of the reconstructed façade model using the developed method is conducted analysing visual performance to assess the completeness and corrections of the experimental results. It is assumed that the human operator can correctly recognise objects in point clouds manually by visual analysis. The completeness and correctness can be predicted by comparing the number of reconstructed façade features with the visible ones from point clouds. Accordingly, the completeness could be defined as the ratio of actually reconstructed façade features amongst all visible reference façade features, while the correctness is defined as the ratio of actual façade features amongst all objects being reconstructed as façade feature. The overall completeness and correctness of each dataset are assessed Table 1 .

Table 1: Evaluation of façade object reconstruction

\begin{tabular}{|l|c|l|}
\hline \multicolumn{1}{|c|}{ Nature } & Dresden & Bonn \\
\hline Observed objects (Ref) & 145 & 93 \\
\hline Reconstructed objects (TP) & 132 & 86 \\
\hline Self-occluded objects (FP) & 2 & 4 \\
\hline Missed objects (FN) & 16 & 21 \\
\hline Completeness (\%) & 89 & 80 \\
\hline Correctness (\%) & 97 & 95 \\
\hline
\end{tabular}

The overall figures of correctness are 97\% (132out of 145) for Dresden and $95 \%$ for Bonn (86 out of 93) while the completeness is about $89 \%$ and $80 \%$. These figures disclose the robustness of the primitive shape integration into façade reconstruction process. The edge orientation analysis for window and door reconstruction seems to be a good solution for removing occlusion successfully. This leads to bring down the overall FP numbers in both datasets. This is because that the method is filtered out occlusions without being recognised as wall objects.

The advantage of detecting only vertical and horizontal lines is that it could help to eliminate the incorrect boundary points detected due to noise. It also leads to obtain a model with correct geometry perceived. For example, as seen in Figure 5b (red box), the irregular boundaries are caused by occluded objects. These parts have been successfully removed from the final model. If all line segments representing boundary points are extracted, then uneven parts, caused by occlusion, present by several skew line segments. This leads to add an additional line regularization step to the geometry reconstruction process. It discarded the holes caused by other objects as shown in Figure 6 (bottom) where a larger area caused by occlusion effect has not been considered for geometric fitting.
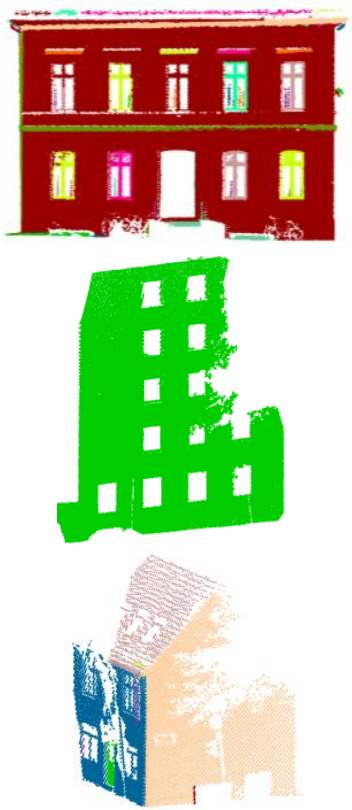
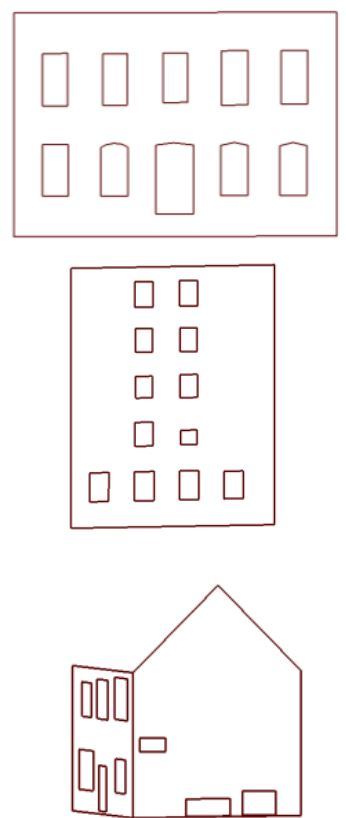

Figure 6: Modelling results of some selected walls (left segmented points, and right - reconstructed models)

\section{CONCLUSION AND FUTURE WORK}

In this paper, we have presented a fully automated approach for the recognition and reconstruction of building façade objects in urban scene. Primitive shapes are introduced to the reconstruction process. Some constraints based on the geometric characteristics of façade objects are applied to remove irrelevant hole-objects. This process has been further enhanced by introducing an edge orientation step. Visual performance illustrated that the proposed approach can be 
applied to reconstruct building façade objects of various shapes with correct topology and geometry compared to real scenes.

Qualitative analysis has been done with regard to two interactively collected reference datasets. The completeness and correctness, as described in our results, are above 95\%, and $80 \%$. This indicates that, in an urban area, majority of façade objects could be modelled accurately using the proposed method. This approach could be used to model not only simple shape object but also more complex shapes.

However, our algorithm may fail when an object is completely occluded by other urban objects as there is a possibility for it could be discarded as a hole by the occlusion effect. Further, some holes, which are not corresponding to façade objects, might generate vertical line segments and those objects are considered as façade objects (FP). Therefore, further enhancement should be taken in future by introducing more constraints. On the other hand, over- and under-segmentation can be seen.

\section{ACKNOWLEDGEMENT}

The authors would like to thank Top-Scan GmbH for providing the MLS data from Bonn, Germany. Thanks also to Mr. Dominik Vock for supporting to acquire MLS data from Münchner Straße, Dresden. The comments and suggestions on this work given by Prof. Hans-Gerd Maas are highly appreciated.

\section{REFERNCES}

Bendels, G. H., Schnabel, R., Klein, R., 2006. Detecting holes in point set surfaces, Journal of WSCG, 14 (2006), 89-96.

Becker S., 2009. Generation and application of rules for quality dependent façade reconstruction. ISPRS Journal of Photogrammetry and Remote Sensing, 64(6), pp. 640-653.

Becker, S., Haala, N., 2007. Refinement of building façades by integrated processing of LIDAR and image data. International Archives of Photogrammetry, Remote Sensing and Spatial Information Sciences 36 (Part 3/W49A), 7-12.

Briese, C., Pfeifer, N., 2008. Line based reconstruction from terrestrial laser scanning data. Journal of Applied Geodesy, 2(2), pp. 85-95 .

Boulaassal, H., Landes, T., Grussenmeyer, P., 2009. Automatic extraction of planar clusters and their contours on building façades recorded by terrestrial laser scanner. International Journal of Architectural Computing, 2009(1), pp. 1-20.

Böhm, J., Becker, S., Haala, N., 2007. Model refinement by integrated processing of laser scanning and photogrammetry. In: Proceedings of the 2nd ISPRS International Workshop 3DARCH (2007).

Douglas, D., Peucker, T., 1973. Algorithms for the reduction of the number of points required to represent a digitized line or its caricature, The Canadian Cartographer 10 (2), 112-122.

Haala, N., Becker, S., Kada, M., 2006. Cell Decomposition for the Generation of Building Models at Multiple Scales. In: IAPRS, Symposium Photogrammetric Computer Vision, vol. 36, part 3, pp. 19-24.
Maas, H.-G., Bienert, A., Scheller, S., Keane, E., 2008. Automatic forest inventory parameter determination from terrestrial laser scanner data. International Journal of Remote Sensing 29 (5), 1579-1593.

Meixner, P., Leberl, F., 2010. Describing buildings by 3dimensional details found in aerial photography. In: The International Archives of the Photogrammetry, Remote Sensing and Spatial Information Sciences, Vienna, Austria, Vol. XXXVIII, Part 7A, pp. 151-156.

Nalani, H. A., Perera, S. N., and Maas, H.-G., 2012. Automatic processing of Mobile Laser Scanner point clouds for building façade detection. In: The International Archives of the Photogrammetry, Remote Sensing and Spatial Information Sciences, Vol. XXXIX-B5, Melbourne, Australia.

Pighin, F., Lewis, J. P., 2007, Practical least-squares for computer graphics. In: Proceedings of the ACM SIGGRAPH 2007 Courses, San Diego, Canada, 1-57.

$\mathrm{Pu}, \mathrm{S}$., 2010. Knowledge based building façade reconstruction from point clouds and images. Ph.D. dissertation, ITC, The Netherlands.

$\mathrm{Pu}$, S., Vosselman, G., 2007. Extracting windows from terrestrial laser scanning. International Archives of Photogrammetry, Remote Sensing and Spatial Information Sciences 36 (Part 3/W52), 320-325.

$\mathrm{Pu}, \mathrm{S}$., Vosselman, G., 2009. Building facade reconstruction by fusing terrestrial laser points and images. Sensors 9(6), pp.4525-4542 .

Ripperda N., 2008. Determination of façade attributes for façade reconstruction. In: The International Archives of the Photogrammetry, Remote Sensing and Spatial Information Sciences, Beijing, China, Vol. XXXVII, Part B3A, pp. 285290.

Reznik S., Mayer H., 2008. Implicit shape models, selfdiagnosis, and model selection for 3D façade interpretation. PFG Photogrammetrie, Fernerkundung, Geoinformation, 2008(3), pp. 187-196.

Schmittwilken J., Plümer L., 2010. Model-based reconstruction and classification of façade parts in 3D point clouds. In: The International Archives of the Photogrammetry, Remote Sensing and Spatial Information Sciences, Saonte-Mande, France, Vol. XXXVIII, Part 3A, pp. 269-274. 